\title{
Performance analysis and evaluation of IEEE 802.11 distributed coordination function using OPNET
}

\author{
Zaynab Mahir Abdel-Ameer ${ }^{1}$, Abdul Kareem A. Najem Alaloosy ${ }^{2}$, Khattab M. Ali Alheeti ${ }^{3}$ \\ ${ }^{1}$ College of Engineering, Computer Engineering Department, AL-Nahrain University Baghdad, Iraq \\ ${ }^{2}$ College of Computer, Computer Networking Systems Department, University of Anbar, Iraq \\ ${ }^{3}$ College of Computer and Information Technology, Computer Networking Systems Department, \\ University of Anbar, Iraq
}

\begin{tabular}{l} 
Article Info \\
\hline Article history: \\
Received Mar 1, 2020 \\
Revised May 14, 2020 \\
Accepted Jun 1, 202 \\
\hline Keywords: \\
Collision avoidance \\
DCF \\
Fragmentation \\
OPNET \\
Throughput
\end{tabular}

Throughput

\begin{abstract}
This paper discusses the distributed coordination function (DCF) access mechanism which is a carrier sense multiple access with collision avoidance (CSMA/CA) scheme. Simulation projects for different DCF performance parameters have been built using the OPNET network simulator. The projects are mainly basic service set (BSS) topology simulated under different parameter values (data rate, fragmentation, RTS/CTS, number of nodes, and load condition). Simulation results show when the DCF access mechanism is better under what load condition, and how to choose the best fragmentation threshold and other access-mechanism specific parameters according to the network conditions. Simulation results were validated against a theoretically calculated maximum throughput (the simulation maximum throughput was about $70 \%$ of the theoretically calculated maximum throughput).
\end{abstract}

This is an open access article under the CC BY-SA license.

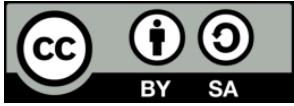

\section{Corresponding Author:}

Khattab M. Ali Alheeti,

Department of Computer Networking Systems,

University of Anbar,

Iraq, Anbar- Ramadi, P.O Box (55431 Baghdad, 55 Ramadi).

Email: co.khattab.alheeti@uoanbar.edu.iq

\section{INTRODUCTION}

Wireless LAN (WLAN) is an adaptable system of data communication applied as an expansion to a wired LAN in a building or campus. Wireless LANs transport and collect data over the air, reducing the requirement for connections of wired. Thus, WLANs integrate data connectivity with user mobility [1,2]. Group of study 802.11 was molded under Project 802 of IEEE to prompt an international standard for WLANs $[3,4]$. The major method to access the medium is named distributed coordination function (DCF) in the 802.11 protocol [5]. It is a random-access method based on (CSMA/CA) protocol. The DCF provides best-effort delivery of data inside a BSS during contention periods. DCF must be implemented in all STAs and can be used in Infrastructure and ad-hoc modes [6, 7]. The MAC layer software also combines a voluntary access scheme named the point coordination function (PCF). Both access schemes are exclusive alternately and work in various time frames identified as the contention period (CP) and contention-free period (CFP) [1]. The DCF defines two methods to use for transmission of a packet [8]. Two-way handshaking is a default method performance named the fundamental access method. This mechanism is described by the current communication of an affirmative acknowledgment by the destination station, upon the effective reception of a packet conveyed by the sender station. Clear transmission of an ACK is necessary since, in the wireless middle, a transmitter cannot define if a packet is effectively obtained by listening to its particular transmission [5]. When two nodes can communicate with another station but cannot communicate 
with each other because of physical or spatial constraints, this situation known as the hidden node problem in wireless networks. The occurrence of hidden nodes may cause degradation performance of the network. It also produces wrong in medium access because the location of the station may indicate a greater privilege of transmission. The basis of the difficulty is that a station applies interference as it misses to sense the presence of transmission from the alternative station and thus believes that the medium is available and accessible for transmission. Carrier sense multiple access with collision detection (CSMA/CD) method, which is exploited in Ethernet networks, mitigates this problem by noticing medium collisions. CSMA/CA method attempt to avert collisions using methods like DCF $[9,10]$.

\section{COORDINATION FUNCTION (802.11 DISTRIBUTED)}

Traffic behavior is considered the main metric for the analysis process for any communication system [11]. Using DCF, for STAs start to transmit they first have to sense the channel for a DIFS time [12]. If the channel is discovered active either directly or through the DIFS, then the station continues to control the channel until it is free during this period. Although the channel is found idle during DIFS, the station still has to generate an arbitrary back off interval before communicating. This procedure will minimize the probability of packet collision and is a collision avoidance (CA) feature of the protocol [13].

The counter of backoff time is decreased while the channel remains inactive and now one of two situations can happen. If the backoff timer reaches zero, the STA starts transmitting, otherwise, if a transmission is noticed on the channel, the timer is frozen and starts to decrement, from the same position, when the channel is sensed idle again after a DIFS. Once a transmission the destination station has to guide a positive acknowledge indicates that the data is well caught since the station is not able to listen to its transmission. However, it has the ability to scalable various data/ control traffic between nodes [14]. The ACK is sent after a SIFS and has priority on the channel since SIFS is the shortest IFS. This way the channel will not be idle for any other station until ACK is received. DCF utilizes a handshaking technique for transmission of the packet. These RTS and CTS frames carry information about the packet length to be transferred. This information can be received by STA in the radius of communication for a sender and a recipient and they can inform their NAV that shown in Figure $1[15,16]$.

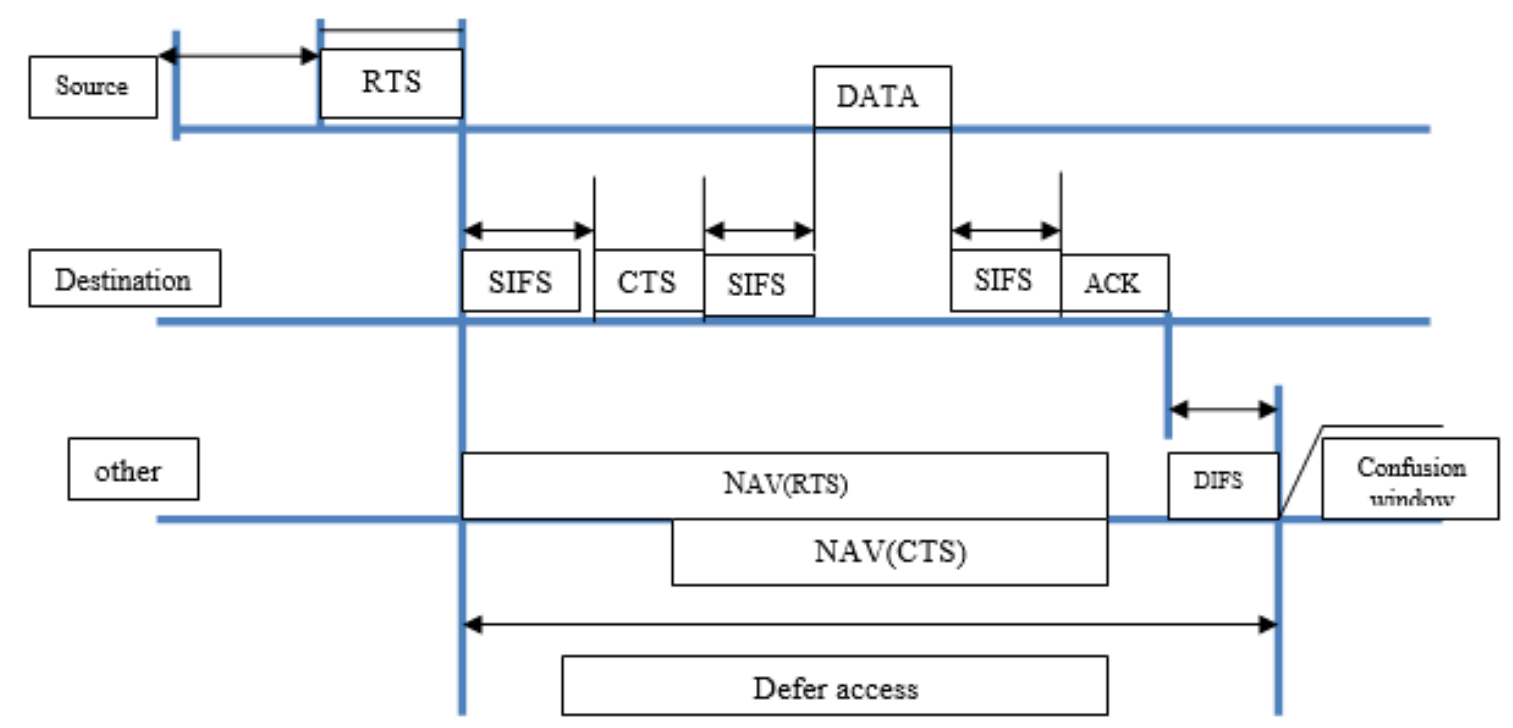

Figure 1. RTS/CTS/data/ACK and NAV setting [15]

RTC/CTS are employed to improve the performance of communication systems [17, 18]. This technique does accurately the same while confirming for a channel. Before transmitting a data packet, an STA directs an RTS frame to the STA destination. If the STA obtains this frame, after a SIFS it replies with a CTS frame. To send the STA data packet, transmitting STA is allowed only. With this technique of collisions straight to the problem of hidden terminals can be avoided since the discovery of one of RTS or CTS frames will avoid the other STA to begin their transmission. However, these mechanisms have a lot of important applications in the modern research area $[19,20]$. 
This four-way handshaking technique has advantages and disadvantages comparing to the basic access method. For instance, if the CTS frame is not received the STA can repeat the whole process in a shorter time than if the ACK frame is not received and the long data frame has to be retransmitted. On the other hand, in the case of smaller BSSs, the amount of the collision will not be so relevant in basic access performance comparing to the additional overheads that the RTS-CTS scheme introduces adding inefficiency to the system [21].

\section{DCF THROUGHPUT}

Medium plays an important role to transfer/ receive control and data [22]. A random backoff method is applied under rules channel access of DCF to decide WLAN station collisions. The random backoff interval is in the unit of a Slot Time, and the arbitrary integer is extracted from an identical distribution throughout the interval $[0, \mathrm{CW}]$, where $\mathrm{CW}$ is the size of the contention window. $\mathrm{CW}$ shall take a primary value of a $\mathrm{CW} \min [23]$. In the state of ineffective communication, $\mathrm{CW}$ is improved to [2*(CW+1)-1]. If $\mathrm{CW}$ successes a max of $\mathrm{CW}$ it shall continue at the value of a max of $\mathrm{CW}$ until it is reset. After an effective acknowledged communication, the value of $\mathrm{CW}$ is reset to a $\mathrm{CW}$ min. DCF services a binary exponential random backoff where the backoff interval $\mathrm{Ib}$ (i) after $i$ ineffective efforts is [21]:

$$
I_{b}(i)= \begin{cases}\frac{2^{i}(a C W \min +1)-1}{2} & 0 \leq i \leq 6 \\ \frac{a C W \max }{2} & i \geq 6\end{cases}
$$

In a quietly network loaded, it would be reasonable to believe that greatest of the stations will effectively achieve channel access after the initial backoff try (i.e., $i=0$ ), so according to (1) above. The average backoff time would be a $\mathrm{CW} \min / 2=\left[31^{*} 20\right] / 2=310 \mu \mathrm{sec}$. Now, the maximum feasible throughput under the DCF channel access method can be computed by (2) [21, 24]:

$$
\begin{aligned}
& \text { Throughput } D C F=(M P D U \text { sizel }[M P D U \text { size }+ \text { MAC header size }]) *(T M P D U, \\
& M A C /[T D I F S+T B a c k \text { off }+T P O H+T M P D U, M A C+T S I F S+T A C K]) * \text { Data Rate }
\end{aligned}
$$

Equation 2 can be illustrated in Figure 2 which shows occupancy times of channel in DCF with the mode of DSSS PHY and 512 bytes size of MPDU. Replacing the values shown in Figure 2 with (data rate=11 Mbps) and (MPDU size=512 bytes) in (2) would result in: Throughput DCF=3.423 Mbps, when using (data rate=2 Mbps) and (MPDU size=512 bytes) in (2) would result in Throughput $\mathrm{DCF}=637.32 \mathrm{Kbps}$, in both cases, the DCF effectiveness is about $31 \%$. Access of DCF channel is highly useless with packet lengths below 1500 bytes.

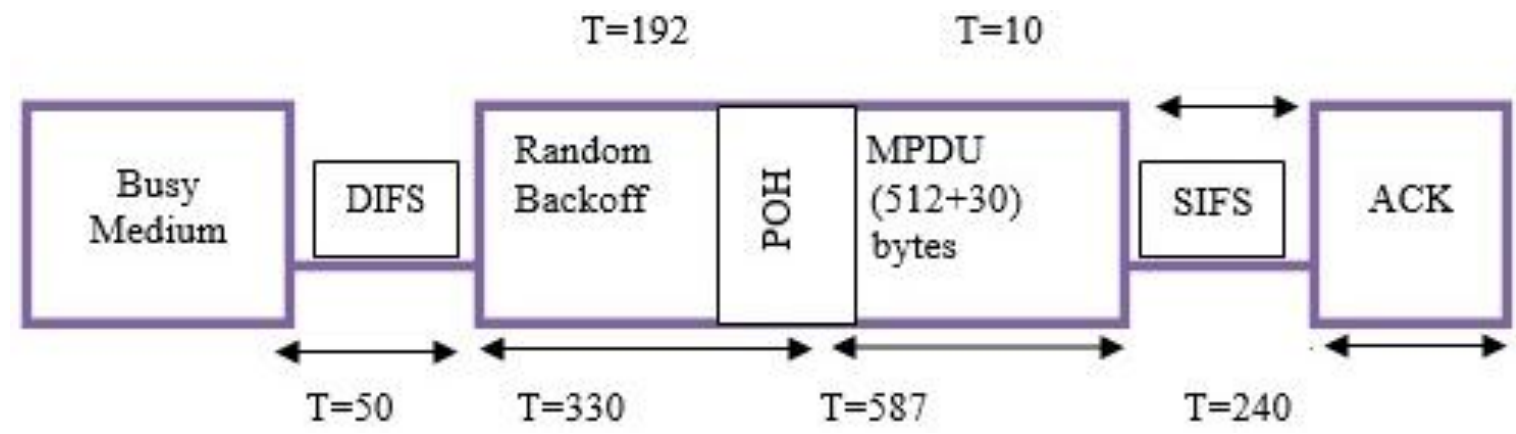

Figure 2. Channel occupancy times under DCF [21]

Using (2) again and putting the (MPDU size $=1500$ bytes) would result in Throughput DCF=6.26 Mbps, in this case, the DCF efficiency is about $57 \%$. It is obvious that for all PHY modes. Throughput success is greater for earlier modes of PHY because the improvidence is mostly because of DIFS/SIFS, average time $\mathrm{f}$ back off, and overheads of PHY, and these parameters are stable and free for the mode of PHY utilized. Table 1 shows the description of IEEE 802.11b PHY parameters. 
Table 1. IEEE 802.11b PHY parameters

\begin{tabular}{|c|c|c|}
\hline Parameter & Duration $(\mu \mathrm{sec})$ & Description \\
\hline Slot time & 20 & Slot time on the channel \\
\hline SIFS & 10 & Short inter-frame space \\
\hline PIFS & 30 & $\mathrm{PIFS}=\mathrm{SIFS}+$ slot time \\
\hline DIFS & 50 & $\mathrm{DIFS}=\mathrm{SIFS}+2 \mathrm{X}$ slot time \\
\hline CWmin & $31 \mathrm{X}$ Slot time & Minimum contention window size \\
\hline CWmax & 1023 X Slot time & Maximum contention window size \\
\hline Average backoff time & $310^{*}$ & $\begin{array}{l}\text { The average duration of the backoff time in a lightly } \\
\text { loaded network }\end{array}$ \\
\hline PHY preamble & 144 & Duration of short and long symbols \\
\hline PHY header & 48 & PHY related information \\
\hline PHY overload (POH) & $192 * *$ & $\mathrm{POH}=\mathrm{PHY}$ Preamble $+\mathrm{PHY}$ header \\
\hline MAC header & $(31 \mathrm{X} 8 \mathrm{bits}) /(11 \mathrm{Mbps})=22.5$ & Assuming 31-byte header at $11 \mathrm{Mbps}$ \\
\hline ACK frame & $(30 \mathrm{X} 8 \mathrm{bits}) /(1 \mathrm{Mbps})=240$ & Ack frame size $=30$ at $1 \mathrm{Mbps}$ \\
\hline Beacon frame & $(71 \mathrm{X} 8$ bits $) /(2 \mathrm{Mbps})=284$ & $\begin{array}{l}\text { Beacon frame size }=61 \text { octets }+ \text { SSID }(\text { service set ID })= \\
71 \text { octets, assuming } 10 \text { bytes, SSID at } 2 \mathrm{Mbps}\end{array}$ \\
\hline Data frame & (MPDUsize X 8bits)/(11 Mbps) & Duration of MPDU at $11 \mathrm{Mbps}$ \\
\hline
\end{tabular}

\section{RESULTS AND DISCUSSION}

The performance metrics are utilized to measure the efficiency of the proposed system. However, before going into the details of the results the collected measures need to be defined [25] which are throughput, delay, retransmission count, and throughput relation. In more details, the throughput metric is the number of bits that sent from wireless LAN layers to higher layers in all nodes of WLAN of the network is referred to as throughput. In addition, the delay metric is a referee to the amount of delay between end to end nodes. Moreover, this metric is end - end delay of the packets achieved by the wireless LAN MACs of all WLAN nodes in the network and sent to the higher layer is represent delay. This delay comprises delay of access of medium at the source MAC, the response of all the fragments separately, and transport of the frames by AP if the access point is allowed. Therefore, retransmission count is the total number for retransmissions of TCP in the network. Inscribed when data is retransmitted from the buffer of TCP unacknowledged.

In this section, we are trying to explain the performance metrics of the proposed system as well as these results will be discussed with the previous studies. Thus, Table 2 shows the throughput results of the DCF project (low load scenarios). Table 3 shows the throughput results of the DCF project (heavy load scenarios). Which (without fragmentation) gives the best throughput and (fragmentation big) gives the worst throughput. From Tables 2 and 3 it can be noticed the following: By comparing Tables 2 and 3 it can be seen that the scenario with a less delay is not essentially producing a better throughput. This is somehow confusing but taking into consideration a DCF technique with RTS method enabled may produce more delay (as the RTS/CTS frames transactions take place) but will give better performance when the collision is avoided. Another explanation may be because that the taken values are average for the whole nodes in the scenario. Table 3 shows very bad and unacceptable delay values that go more than $1 \mathrm{~min}$. This shows that it is not useful to apply DCF with rates of data at $2 \mathrm{Mbps}$ or $11 \mathrm{Mbps}$ for applications of multimedia as the number of applied traffic packets will be more than what can be controlled. In this state, the buffer will be full and the number of dropped packets rises.

The throughput, data rate, and the number of nodes are shown in Table 2. For this, it displays that the throughput is monotonically increasing with the number of nodes. By examining each column of Table 2 it can be noted that the average throughput rises when nodes number goes up. This may be supported as further nodes refer to further traffic, which in turn raises the throughput as long as the network connection capacity has not been applied. Since Table 2 displays only the low load scenarios average throughput (the max. throughput is about $2 \mathrm{Kbps}$ ), there is no big difference between the $2 \mathrm{Mbps}$ results and the corresponding $11 \mathrm{Mbps}$ results. This is not true for Table 3 results which show a big difference between the average throughput of $2 \mathrm{Mbps}$ results and the corresponding $11 \mathrm{Mbps}$ results (about three to six times better). As calculated that the maximum throughput for $2 \mathrm{Mbps}$ is $(637.32 \mathrm{Kbps})$, and for $11 \mathrm{Mbps}$ is $(3.423$ Mbps). In the low load case, the traffic was $(2 \mathrm{Kbps})$ much below the maximum achievable throughput by both rates; that is why there is no difference between the two rates results. While in the heavy load case the traffic was due to the multimedia profile which can be more than $5 \mathrm{Mbps}$. In this case, the $2 \mathrm{Mbps}$ rate made a maximum throughput of about $(450 \mathrm{Kbps})$ (which is quite reasonable since it is about $70 \%$ of the maximum perfect channel theoretical throughput). The $11 \mathrm{Mbps}$ rate made a maximum throughput of about $(2.33 \mathrm{Mbps}$ ) (which is quite reasonable since it is about $68 \%$ of the maximum perfect channel theoretical throughput).

However, throughput delay and fragmentation are demonstrated in Table 2 and Table 3 . In addition, low load and heavy load are noticed in both Tables. The best throughput results were when fragmentation was disabled. While most of the worst throughputs in the low load case are when fragmentation, 
the threshold set to big (512 bytes) as shown in Table 2. Table 3 shows the reverse; as all of the worst throughputs in the heavy load case is when the fragmentation threshold set to small (128 bytes). This can be validated by the fact that fragmentation is to enhance the performance when many retransmissions are taking place. In lightly loaded network fragmentation is no more than additional overhead that degrades the performance. This is why the (without fragmentation scenarios gave the best performance) in Tables 2 and 3. Also in the heavily loaded network when there is no or very small error rate, fragmentation is an unwanted additional overhead especially (small fragmentation) when almost all packets are fragmented into smaller packets thus the network is flooded with overhead. This is why all of the worst throughputs in the heavy load case are when the fragmentation threshold set to small (128 bytes). In general, the fragmentation threshold must be modified according to the load, rate of bit error, and nodes number of the network. Since delay change against the fragmentation used, it can be observed that the fragmentations raise the delay specifically the small threshold of fragmentation. And this rise in delay because of fragmentation is much in the minor rates of data at (2 Mbps) than it is in the high rates of data at (11 Mbps).

Table 2. DCF low load scenarios average delay (msec)

\begin{tabular}{|c|c|c|c|c|c|c|c|c|c|}
\hline \multirow[b]{2}{*}{$\begin{array}{l}\text { No. of PCs } \\
\& \text { data Rate }\end{array}$} & \multicolumn{3}{|c|}{ Without fragmentation } & \multicolumn{3}{|c|}{ Fragmentation big (512 bytes) } & \multicolumn{3}{|c|}{ Fragmentation small (128 bytes) } \\
\hline & $\begin{array}{l}\text { Without } \\
\text { RTS }\end{array}$ & RTS big & $\begin{array}{l}\text { RTS } \\
\text { small }\end{array}$ & $\begin{array}{l}\text { Without } \\
\text { RTS }\end{array}$ & RTS big & $\begin{array}{l}\text { RTS } \\
\text { small }\end{array}$ & $\begin{array}{l}\text { Without } \\
\text { RTS }\end{array}$ & RTS big & $\begin{array}{l}\text { RTS } \\
\text { small }\end{array}$ \\
\hline 2 PCs-2 Mbps & 3.1 & 3.2 & 3.5 & 2.8 & 4.0 & 4.2 & 4.8 & 4.7 & 5.1 \\
\hline 4 PCs-2 Mbps & 4.0 & 5.2 & 5.3 & 5.1 & 4.9 & 6.4 & 7.7 & 7.1 & 8.0 \\
\hline $4 \mathrm{PCs}-11 \mathrm{Mbps}$ & 2.9 & 2.5 & 3.0 & 2.7 & 2.9 & 3.3 & 4.8 & 4.7 & 5.5 \\
\hline 8 PCs-2 Mbps & 6.2 & 8.8 & 6.9 & 8.8 & 6.1 & 6.8 & 8.6 & 8.7 & 10.3 \\
\hline 16 PCs-11 Mbps & 3.5 & 4.0 & 6.2 & 4.2 & 4.2 & 4.7 & 6.4 & 6.8 & 7.8 \\
\hline
\end{tabular}

Table 3. DCF heavy load scenarios average delay (sec)

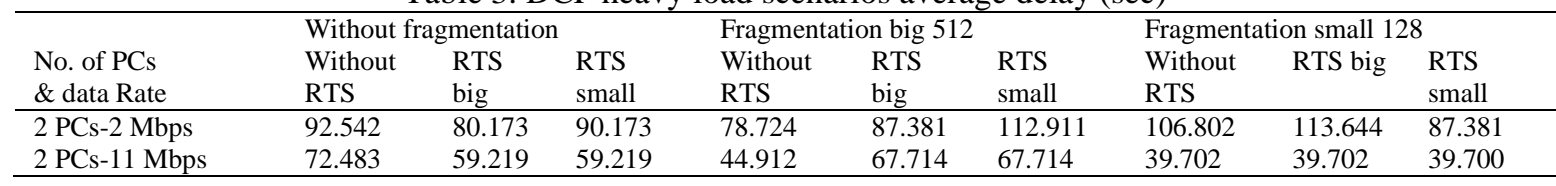

The RTS and throughput delay is illustrated in Table 2. However, it improves the implementation as the best of the throughputs are under the small RTS column, while RTS and RTS outcomes were near to each other. This can be confirmed as RTS is a collision avoidance mechanism for the DCF technique and also because of the low load condition RTS small is giving the best performance. In Table 3, the RTS effect is not clear because of the limited number of simulated scenarios in the heavy load case. Since delay change against the RTS threshold employed as shown in Tables 2 and 3, it can be observed that RTS rises by using the delay especially the small threshold of RTS. And this rise in delay suitable to consuming RTS is much in the minor rates of data at $2 \mathrm{Mbps}$ than it is in the high rates of data at $11 \mathrm{Mbps}$. The effect of load on the behavior is comparing the first two rows of Tables 2 and 3 show that:

- $11 \mathrm{Mbps}$ rate is better than $2 \mathrm{Mbps}$ only in the heavy load condition.

- Fragmentation small reduces the performance in the serious load and low rate of bit error conditions.

\section{CONCLUSION}

According to the results and discussion, the following conclusions can be made. First, it is not useful to exploit DCF with rates of data at $2 \mathrm{Mbps}$ or $11 \mathrm{Mbps}$ for applications of multimedia as the number of produced traffic packets will be more than what can be recovered. In this state, the buffer will be full and dropped packets number rises exponentially. Second, DCF simulation in OPNET produces a maximum throughput of about $70 \%$ of the maximum perfect channel theoretical throughput. This is to be considered near to the practical maximum throughput. Third, in DCF, the heavily loaded network when there is no, or very small error rate, fragmentation is an unwanted additional overhead especially (small fragmentation) when almost all packets are fragmented into smaller packets thus the network is flooded with overhead. Fourth, according to the load, the threshold of fragmentation should be modified, rate of bit error, and nodes number, of the network. Where a small threshold of fragmentation reduces DCF performance with serious load, and rate conditions of low bit error. 


\section{REFERENCES}

[1] R. Jordan and C. T. Abdallah, "Wireless communications and networking: an overview," in IEEE Antennas and Propagation Magazine, vol. 44, no. 1, pp. 185-193, 2002.

[2] A. K. Salkintzis, "Interworking techniques and architectures for WLAN/3G integration toward 4G mobile data networks," in IEEE Wireless Communications, vol. 11, no. 3, pp. 50-61, 2004.

[3] G. Bianchi, "Performance analysis of the IEEE 802.11 distributed coordination function," in IEEE Journal on Selected Areas in Communications, vol. 18, no. 3, pp. 535-547, 2000.

[4] S. Banerji, "Upcoming standards in wireless local area networks," Wireless and Mobile Technologies, vol. 1, no 1, pp. 6-11, 2013.

[5] I. Tinnirello, G. Bianchi, and Y. Xiao, "Refinements on IEEE 802.11 distributed coordination function modeling approaches," in IEEE Transactions on Vehicular Technology, vol. 59, no. 3, pp. 1055-1067, 2009.

[6] R. P. de Almeida Martins, "IEEE 802.11b WLAN performance measurements and optimization," Master Thesis, Department of Communication Technology, Aalborg University, June 2004.

[7] N. Cranley and L. Murphy, "Handbook of research on wireless multimedia: Quality of service and solutions," Liam Murphy (Ed.), Information Science Reference, IGI Global, 2008,

[8] A. Balador, M. Ghasemivand, A. Movaghar, and S. Jabbehdar, "An adaptive contention window control for improving DCF throughput and fairness," European J. Scientific Research, vol. 45, no. 2, pp. 310-323, 2010.

[9] H-H. Choi, J-M. Moon, I-H. Lee, and H. Lee, "Carrier sense multiple access with collision resolution," IEEE Communications Letters, vol. 17, no. 6, pp. 1284-1290, 2013.

[10] S. Rahman, "Throughput analysis of IEEE 802.11 distributed coordination function in presence of hidden stations," Stanford Technical Report, Department of Electrical Engineering, Stanford University, 2003.

[11] R. Lara-Cueva and M. Simba-Herrera, "Performance evaluation for outdoor wireless scenarios based on IEEE $802.11 \mathrm{~b} / \mathrm{g}$ in the presence of co-channel interference," Bulletin of Electrical Engineering and Informatics, vol. 9 , no.1, pp. 187-197, 2020.

[12] A. Goldsmith, "Wireless communications," Cambridge: Cambridge University Press, 2005.

[13] S. Suhane, S. Sharma, and V. Sharma, "Performance analysis of backoff algorithm in IEEE 802.11 networks," International Journal of Scientific \& Engineering Research, vol. 2, no. 6, pp. 1-5, 2011.

[14] A. Yonis, "Influence of low power consumption on IEEE 802.15. 4 in wireless networks performance," Bulletin of Electrical Engineering and Informatics, vol.9, no. 1, pp. 205-211, 2020.

[15] B. Keegan and M. Davis, "An experimental analysis of the call capacity of IEEE $802.11 \mathrm{~b}$ wireless local area networks for VoIP telephony," In Irish Signals and Systems Conference, Dublin, pp. 283-287, 2006.

[16] T. Shigeyasu and H. Matsuno, "Effect of adaptive RTS/CTS ON/OFF control method for mitigating transmission overhead of IEEE 802.11DCF," In 2010 IEEE International Conference on Broadband, Wireless Computing, Communication and Applications, pp. 627-632, 2010.

[17] K. I. Patel and M. M. Vegad, "Effectiveness of RTS/CTS in IEEE 802.11 wireless adhoc network in light of order dependent capture," In 2013 IEEE Fourth International Conference on Computing, Communications and Networking Technologies (ICCCNT), pp. 1-6, 2013.

[18] W-P. Nwadiugwu and D-S. Kim, "Novel MIMO-UWB based RTS/CTS collaboration with scalable rate prediction for industrial WSNs," In 2019 15th IEEE International Workshop on Factory Communication Systems (WFCS), pp. 1-4, 2019.

[19] Z. Na, L. Ningqing, and Q. Yu, "Improved RTS-CTS algorithm to solve mobile hidden station problem in MANET," In Proceedings of IEEE 2011 Cross Strait Quad-Regional Radio Science and Wireless Technology Conference, vol. 1, pp. 812-815, 2011.

[20] G. Habib and C. Bassil, "Influence of the RTS/CTS in VANET," In 2013 IEEE 13th Mediterranean Microwave Symposium (MMS), pp. 1-4, 2013.

[21] N. Wattanamongkhol, W. Srichavengsup, S. Nakpeerayuth, and L. Wuttisiittikulkij, "Performance analysis of modified backoff algorithm in IEEE 802.11 networks," In 2007 3rd IEEE/IFIP International Conference in Central Asia on Internet, Tashkent, pp. 1-5, 2007.

[22] A. Butar-Butar, J. Leong, and M. Irwanto, "Effect of DC voltage source on the voltage and current of transmitter and receiver coil of $2.5 \mathrm{kHz}$ wireless power transfer," Bulletin of Electrical Engineering and Informatics, vol. 9 , no. 2, pp. 484-491, 2020.

[23] R. Papa, A. Galderisi, M. C. Vigo Majello, and E. Saretta, "Smart and resilient cities. A systemic approach for developing cross-sectoral strategies in the face of climate change," TeMA Journal of Land Use, Mobility and Environment, vol. 8, no. 1, pp. 19-49, 2015.

[24] A. Duda, "Understanding the performance of 802.11 networks," In 2008 IEEE 19th International Symposium on Personal, Indoor and Mobile Radio Communications, Cannes, vol. 8, pp. 1-6, 2008.

[25] D. Vassis, G. Kormentzas, A. Rouskas, and I. Maglogiannis, "The IEEE 802.11g standard for high data rate WLANs, "IEEE Network, vol. 19, no. 3, pp. 21-26, 2005. 